\title{
PD-LI Predicts Poor Prognosis in Surgically Resected Limited Stage Small-Cell Lung Cancer
}

This article was published in the following Dove Press journal:

Cancer Management and Research

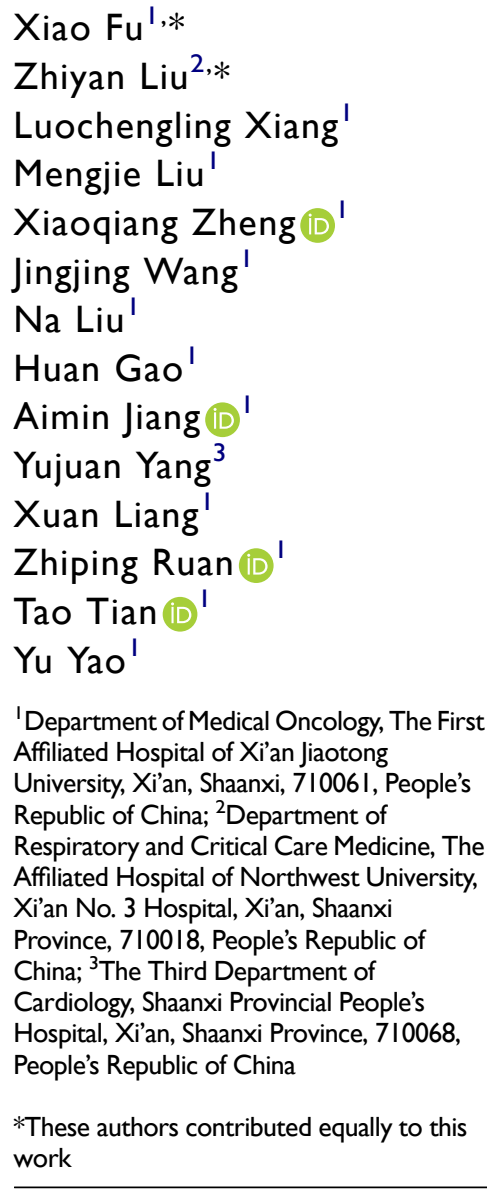

Correspondence: Tao Tian

Department of Medical Oncology, The First Affiliated Hospital of Xi'an Jiaotong University, No. 277 Yanta West Road, Xi'an, Shaanxi, People's Republic of China

Tel $+86 \quad 13572206784$

Fax +862985324086

Email tiantao0607@I63.com

Yu Yao

Department of Medical Oncology, The First Affiliated Hospital of Xi'an Jiaotong University, No. 277 Yanta West Road, Xi'an, Shaanxi, People's Republic of China

Tel +86 I3572 I0 I6I I

Fax +862985324086

Email yaoyu 123@xjtufh.edu.cn
Purpose: Small-cell lung cancer (SCLC) is an aggressive high-grade neuroendocrine tumor with limited treatment strategies. Programmed death 1 (PD-1) and its ligand (PD-L1), deltalike ligand-3 (DLL-3), and poly ADP-ribose polymerase (PARP) inhibitors have shed light on the treatment of extensive stage-SCLC. However, the expression and prognostic role of PD-L1, DLL-3, and PARP are barely explored in surgically resected limited stage-SCLC (LS-SCLC).

Methods: We retrospectively reviewed 404 SCLC patients from 2011 to 2018 in the First Affiliated Hospital of Xi' an Jiaotong University and collected 43 surgically resected LS-SCLC samples with adequate materials and histological specimens containing abundant tumor cells. Immunohistochemistry staining of PD-L1, DLL-3, and PAPR1 was performed by anti-PD-L1 (22C3/Dako), anti-DLL-3, and anti-PAPR1 antibodies, respectively. Positive expression of PD-L1 was characterized as $>5 \%$ tumor cells and/or tumor-infiltrating immune cells expressing PD-L1. The correlation between PD-L1, DLL-3, PARP1, and clinicopathological characteristics of surgically resected LS-SCLC patients was performed by $\chi^{2}$ test. The survival curves were calculated by the Kaplan-Meier method and analyzed by the Log rank test and Cox proportional hazards model.

Results and Conclusion: $63.04 \%$ patients were positive for PD-L1, 65.12\% were positive for DLL-3, and $20.93 \%$ were positive for PARP1. DLL-3 was significantly overexpressed in SCLC tissues, compared with matched para-noncancerous tissues. Male, elder than 60 years old, advanced TNM stage, smoking, and positive PD-L1 expression predicted shorter DFS, while patients received adjuvant therapy performed better DFS. Further multivariate analysis revealed that TNM stage $(\mathrm{HR}=2.51,95 \% \mathrm{CI}=1.31-4.78, P=0.005)$ was an individual prognostic factor for DFS in LS-SCLC. Moreover, advanced TNM stage and positive PD-L1 expression also indicated worse OS, but adjuvant therapy improved OS in LS-SCLC. Multivariate analysis demonstrated that PD-L1 and TNM stage were independent and significant negative predictive factors for $\mathrm{OS}(\mathrm{HR}=2.89,95 \% \mathrm{CI}=1.21-6.93, P=0.017$; $\mathrm{HR}=2.49,95 \% \mathrm{CI}=1.25-4.94, P=0.009$ for PD-L1 and TNM stage, respectively), while adjuvant treatment was an independent positive prognostic factor for OS (HR $=0.37,95 \%$ $\mathrm{CI}=0.17-0.81, P=0.012$ ).

Keywords: surgically resected LS-SCLC, PD-L1, DLL-3, PARP1

\section{Introduction}

Small-cell lung cancer (SCLC) is an aggressive high-grade neuroendocrine tumor with limited treatment strategies. Although SCLC is sensitive to chemoradiotherapy, it has a high relapse rate with a dismal prognosis. Chemotherapy combined anti-PD-L1 has been approved for the first line treatment of extensive stage-SCLC (ES-SCLC). ${ }^{1}$ Subgroup of ES-SCLC with positive PD-L1 expression $(\geq 1 \%)$ 
seemed to have a better response rate and survival, indicating that PD-L1 may predict efficacy to immunotherapy and survival in SCLC.

DLL-3 is an inhibitory ligand to Notch pathway, which is involved in the development of pulmonary neuroendocrine cells. ${ }^{2}$ DLL-3 is upregulated and overexpressed on the cell surface in SCLC and other high-grade neuroendocrine tumors ${ }^{3,4}$ and predicted the response of Rova- $\mathrm{T}$ (NCT01901653). ${ }^{5}$

PARP is a critical enzyme involved in the process of DNA repair, and catalyzes the synthesis of poly (ADPribose) (PAR) chain after recognition of single-strand DNA breaks and prevents cells from apoptosis by cleaving DNAdamage and thus inhibits apoptosis induction. The safety and efficacy have been tested in several clinical trials, indicating that PARP1 inhibitors with or without conventional chemotherapy were well-tolerated and effective in ES-SCLC. ${ }^{6-8}$

With the success in ES-SCLC, more attention needs to be paid to immunotherapy and targeted therapy in LS-SCLC. Whether PD-1/PD-L1 antibodies, DLL-3, and PAPR1 inhibitors could be used in LS-SCLC is still unknown. Understanding the expression of PD-L1, DLL-3, and PARP1 in LS-SCLC and their correction to clinicopathological features and outcomes in LS-SCLC may facilitate the development of new strategies in LS-SCLC.

\section{Patients and Methods}

\section{Patients}

We retrospectively screened 404 SCLC patients diagnosed with SCLC from January 2011 to January 2018 in the First Affiliated Hospital of Xi'an Jiaotong University. 46 of them underwent surgery, and adequate materials and histological specimens containing abundant tumor cells were available for 43 of them. All protocols were approved by the Ethics Committee of Xi'an Jiaotong University, and informed consent was obtained from all patients before surgery. Our study complied with the Declaration of Helsinki. Upon enrollment, SCLC was confirmed in all patients by biopsy or surgical pathology and graded by the American Joint Committee on Cancer (AJCC) 8th tumor lymph node metastasis (TNM) staging system. The clinicopathological characteristics of the patients were collected from the hospital medical records, and registered patients were prospectively followed up until July 1, 2020 .

The clinicopathological characteristics of 43 surgically resected LS-SCLC are shown in Table 1. The medium age is 60 years, and ranges from $28-79$ years. Thirty-five $(81.40 \%)$ of them were male. Current or former smokers accounted for $62.80 \%$. Twenty-seven patients $(62.79 \%)$ were central SCLC, others were peripheral SCLC. NSE was overexpressed in 27 patients $(62.79 \%)$ before surgery. According to TNM 8th version, 14 patients (32.56\%) were at stage I, 23 patients $(53.49 \%)$ were stage II, and 6 patients $(13.95 \%)$ were stage III.

\section{Immunohistochemistry (IHC) Staining}

The expression of PD-L1 (DAKO, 22C3), DLL-3 (bs7860R, Bioss, China), ASCL-1 (bs-1155R, Bioss, China), PARP1 (bs-20764R, Bioss, China), and SLFN11 (sc374,339 , Santa Cruz, US) were detected in $5-\mu \mathrm{m}$ sections cut from FFPE SCLC tissues by IHC as previously described. ${ }^{9}$ Immunostaining for PD-L1 was defined positive if it was observed in the membrane and/or cytoplasm in $>5 \%$ of the tumor cells and tumor infiltrating immune cells. $^{10}$

Table I The Characteristics of Forty-Three Surgically Resected LS-SCLC

\begin{tabular}{|c|c|c|c|}
\hline Characteristics & Patients $(n=43), n(\%)$ & Characteristics & Patients (n=43), n (\%) \\
\hline Age & & Yes & $24(55.81 \%)$ \\
\hline Medium (years) & 60 & Central or Peripheral & \\
\hline Range (years) & $28-79$ & Central & 27 (62.79\%) \\
\hline Gender & & Peripheral & $16(37.21 \%)$ \\
\hline Male & $35(81.40 \%)$ & NSE level & \\
\hline Female & $8(18.60 \%)$ & NSE normal & $2(4.65 \%)$ \\
\hline \multirow[t]{2}{*}{ Smoking status } & & NSE high & 27 (62.79\%) \\
\hline & & NSE unknown & $14(32.56 \%)$ \\
\hline Smoker (current or former) & 27 (62.80\%) & TNM Stage & \\
\hline Non-smoker & $16(37.20 \%)$ & $\mathrm{I}$ & $14(32.56 \%)$ \\
\hline Onset symptom & & ॥ & $23(53.49 \%)$ \\
\hline No & 19 (44.19\%) & III & $6(13.95 \%)$ \\
\hline
\end{tabular}




\section{Statistical Analysis}

The association between PD-L1, DLL-3, ASCL-1, PARP1, SLFN11, and clinicopathological characteristics of LS-SCLC patients was performed by $\chi^{2}$ or student's$t$-test, whenever is appropriate. DFS was defined as the time from operation until the first documented progression of disease, death, or date of last follow-up. OS was defined as initial diagnosis until the date of death or last follow-up. The survival curves were calculated by the Kaplan-Meier method and analyzed by the Log rank test. Univariate and multivariate regression was performed by the Cox proportional hazards model. All variables that had $P$-values of less than 0.05 were included in the Cox model. All tests were two-sided, and $P$-values of less than 0.05 was considered statistically significant. All of the statistical analyses were conducted using SPSS22.0 (Chicago, IL, US).

\section{Results}

The Expression of PD-LI, DLL-3, PARPI, ASCL-I, SLFNII, and Correlation to Clinicopathological Factors

PD-L1, DLL-3, PARP1, ASCL-1, and SLFN11 were examined in 43 samples and the representative images were shown in Figure 1 and Supplementary Figure 1. Among 43 surgically resected LS-SCLC specimen, PDL1 was positive $(>5 \%)$ in $58.14 \%$ ( 25 out of 43 ) cases. PAPR1 was not significantly different between tumor and matched non-cancerous tissues (Figure 1B), which was only positive in nine patients $(20.93 \%)$. DLL-3 was significantly overexpressed in tumor tissues, compared with matched para-noncancerous tissues (Figure 1C). And the positive rate was $65.12 \%$ (28/43), similar to previously reported. No significant relationship was found between PD-L1, PARP1, or DLL-3 and clinicopathological factors (Table 2).

In the PARP1 pathway, SLFN11 is actively recruited to sites of DNA damage, inhibits homologous recombination, ${ }^{11}$ and activates a cellular replication-stress response. SLFN11 expression correlates with sensitivity to DNA-damaging agents. ${ }^{12}$ In our cohort, SLFN11 was not significantly different between tumor and matched noncancerous tissues, and 20 patients (46.51\%) positively expressed SLFN11 (Supplementary Figure 1A). Achaetescute homolog-1 (ASCL-1) transcriptionally activates DLL-3 and is also upregulated in neuroendocrine tumor, including SCLC, and has been shown to be positively related to DLL-3 in surgically resected SCLC samples. ${ }^{13}$ ASCL-1 was also upregulated in SCLC (Supplementary Figure 1B), compared with matched para-noncancerous tissues, and was positively expressed in 29 patients (67.44\%). Moreover, no significant relationship was found between ASCL-1, SLFN11, and clinicopathological factors (Supplementary Table 1).

\section{Relationship Between PD-LI, DLL-3, PARPI, ASCL-I, and SLFNII}

A positive correlation has been demonstrated in previous studies, however, we did not find a correlation between DLL-3 and ASCL-1 in our cohort $(\mathrm{r}=0.12, P=0.23)$. But we noticed a positive correlation between PARP1 and SLFN11 $(\mathrm{r}=0.21, P=0.09)$, though it was not statistically significant.

We also testified the relationship between PD-L1, DLL-3, and PAPR1, but no correlation was found between PD-L1, DLL-3, and PAPR1: $\mathrm{r}=0.01, P=0.94$ for PD-L1 and DLL-3; $\mathrm{r}=-0.01, P=0.96$ for PD-L1 and PAPR1; $\mathrm{r}=$ $-0.10, P=0.50$ for DLL-3 and PARP1.

\section{Gender, Age, Smoking Status, Adjuvant Treatment, TNM Stage, and PD-LI Expression Were Correlated with DFS}

The median duration of follow-up was 30 months (range from 0.2-87 months). Among clinicopathological characters, we found that male patients had poorer DFS than female patients (mDFS: $22.00 \pm 2.90$ vs $29.00 \pm 33.94$ months in male and female patients, respectively, $P=0.031$, Figure $2 \mathrm{~A}$ ). Elder patients (elder than 60 years old) performed poorer DFS than those who were less than 60 years old (mDFS: $12.00 \pm 5.59$ vs $33.00 \pm 11.80$ months in elder and younger than 60 years old patients, respectively, $P=0.018$, Figure 2B). Adjuvant therapy (either chemotherapy or radiotherapy) improved DFS (mDFS: $29.00 \pm 8.90$ vs $21.00 \pm 5.03$ months in patients with or without adjuvant therapy, respectively, $P=0.018$, Figure 2C). Advanced TNM stage also correlated with poor DFS (mDFS: $29.00 \pm 8.42,22.00 \pm 0.79$, and $1.00 \pm 0.00$ months in TNM stage I-III, respectively, $P=0.000$, Figure 2D). Current or former smokers seemed to perform shorter DFS (mDFS: $21.00 \pm 7.75$ vs $29.00 \pm 11.00$ months in current or former smokers and never smokers respectively, $P=0.053$, Figure $2 \mathrm{E}$ ). We further analyzed the relationship between smoking index and prognosis, and noticed that moderate smokers had the worst DFS (mDFS: 29.00 $\pm 11.00,6.00 \pm 3.00$, and 21.00 \pm 4.79 months in never smokers, moderate smokers, and heavy smokers, respectively, $P=0.021$, Figure $2 \mathrm{~F}$ ). While central or 

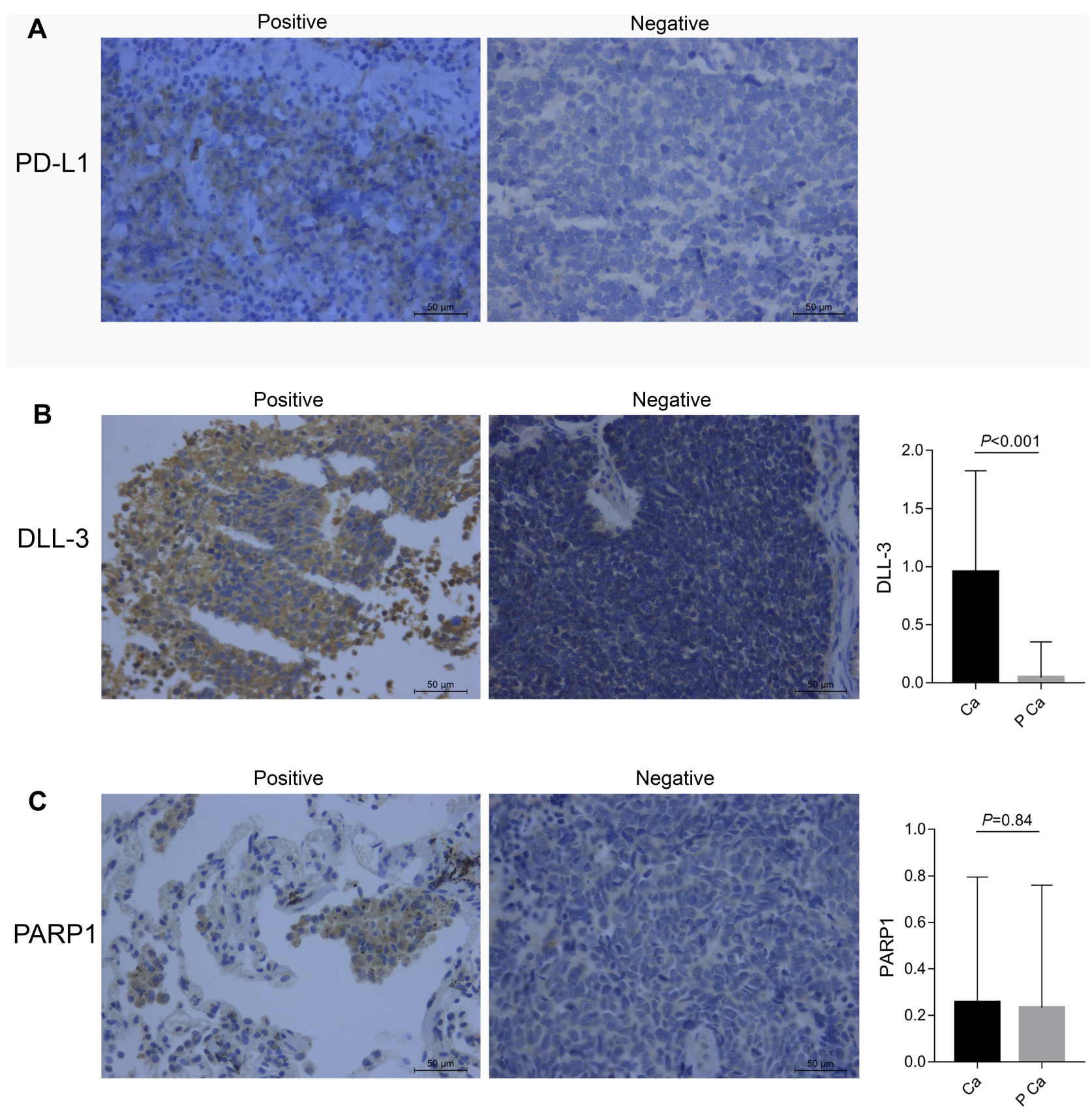

Figure I The distribution of PD-LI (A), DDL-3 (B), and PARPI (C).

peripheral tumor, comorbidities, onset symptoms, or NSE level did not have significant effects on LS-SCLC prognosis (Supplementary Figure 2A-D).

Patients with positive PD-L1 expression had significantly worse DFS than those with negative PD-L1 (mDFS: 21.00 \pm 3.50 vs $34.00 \pm 29.70$ months in PD-L1 positive and negative groups respectively, $P=0.049$, Figure $3 \mathrm{~A})$. Neither PARP1, DLL-3, ASCL-1, nor SLFN11 preformed any difference in DFS (Figure 3B and C, Supplementary Figure 3A and $\underline{C}$ ).
Univariate analysis demonstrated that gender $(\mathrm{HR}=0.36 .95 \% \mathrm{CI}=0.14-0.96, P=0.004)$, age $(\mathrm{HR}=2.11$, $95 \% \mathrm{CI}=1.11-4.03, P=0.024)$, TNM stage $(\mathrm{HR}=2.22,95 \%$ $\mathrm{CI}=1.21-4.07, \quad P=0.010), \quad$ and adjuvant treatment $(\mathrm{HR}=0.44,95 \% \mathrm{CI}=.21-0.90, P=0.025)$ are associated with DFS, and further multivariate analysis identified TNM stage $(\mathrm{HR}=2.51,95 \% \mathrm{CI}=1.31-4.78, P=0.005)$ as an individual prognostic factor for DFS in LS-SCLC (Table 3). 


\begin{tabular}{|c|c|c|c|c|c|c|c|c|c|c|c|}
\hline$a$ & 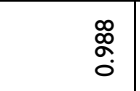 & $\begin{array}{l}0 \\
\stackrel{0}{\infty} \\
0 \\
0\end{array}$ & & @ & \begin{tabular}{l|} 
\\
$\stackrel{\infty}{0}$ \\
$\stackrel{0}{0}$
\end{tabular} & $\begin{array}{l}\text { वें } \\
\text { ते }\end{array}$ & $\begin{array}{l}\bar{m} \\
\stackrel{n}{o}\end{array}$ & $\begin{array}{l}8 \\
\stackrel{2}{0} \\
0\end{array}$ & 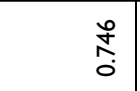 & స్్ㅁ & 命 \\
\hline$x_{x}^{*}$ & : & $\begin{array}{l}0 \\
\stackrel{0}{0} \\
0 \\
0\end{array}$ & & $\frac{m}{0}$ & స్ & 을 & 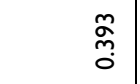 & $\frac{\infty}{\frac{\infty}{0}}$ & $\frac{n}{0}$ & $\stackrel{m}{m}$ & $\underset{\infty}{\bar{\infty}}$ \\
\hline 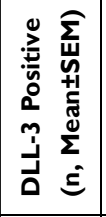 & $\underline{\underline{n}} \underline{m}$ & $\tilde{N}$ in & $=$ & $\simeq$ & 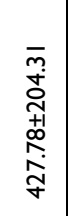 & \pm \pm & \pm \pm & $\leq=$ & 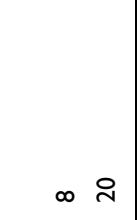 & N 0 & $=\underline{m} \sigma$ \\
\hline 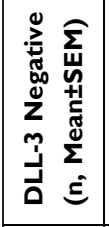 & $\infty \wedge$ & $\simeq \mathrm{m}$ & n & 으 &  & in 으 & $a 0$ & 은 & n 0 & $0=$ & $m \subseteq N$ \\
\hline$a$ & $\underset{\substack{m \\
0}}{0}$ & $\begin{array}{l}\stackrel{q}{t} \\
\hat{0}\end{array}$ & & 융 & $\frac{\dot{m}}{0}$ & $\frac{\wp}{f}$ & $\frac{\Omega}{0}$ & $\begin{array}{l}\hat{\infty} \\
\stackrel{0}{0} \\
0\end{array}$ & $\begin{array}{c}\substack{0 \\
\infty \\
0 \\
0} \\
0\end{array}$ & $\underset{\substack{0 \\
\text { o. } \\
0}}{ }$ & 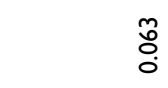 \\
\hline ते & $\begin{array}{l}\stackrel{n}{\alpha} \\
\hat{\sigma}\end{array}$ & $\begin{array}{l}\infty \\
\vdots \\
0 \\
0\end{array}$ & & ợ & $\stackrel{\widehat{N}}{\underline{\Lambda}}$ & 欱 & $m \stackrel{\stackrel{\leftrightarrow}{a}}{\stackrel{\infty}{-}}$ & $\begin{array}{c}\tilde{o} \\
\hat{0}\end{array}$ & $\begin{array}{l}\text { 공 } \\
\text { O }\end{array}$ & 荅 & $\frac{a}{\mathcal{n}}$ \\
\hline  & $\circ \mathrm{m}$ & $\sim \sim$ & t & n &  & in $t$ & $m o$ & $\circ \mathrm{m}$ & 은 & $m o$ & $0 \wedge r$ \\
\hline 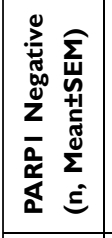 & $\simeq=$ & 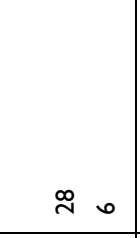 & $\approx$ & $=$ & $\begin{array}{c}\tilde{c} \\
\stackrel{\tilde{m}}{m} \\
\frac{m}{+1} \\
\stackrel{m}{m} \\
\frac{m}{0}\end{array}$ & 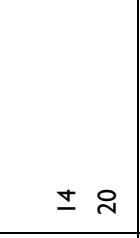 & $\stackrel{N}{ \pm}$ & $\bar{N} \underline{m}$ & $\underline{m}$ & 으 헌 &  \\
\hline$a$ & 怘 & 望 & & ‡. & $\begin{array}{l}\mathfrak{n} \\
\hat{f} \\
0\end{array}$ & ণ্ণ & $\begin{array}{c}\substack{\infty \\
\infty \\
0} \\
\end{array}$ & 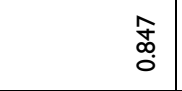 & $\frac{8}{0}$ & $\begin{array}{l}\bar{o} \\
\stackrel{0}{0} \\
0\end{array}$ &  \\
\hline${ }^{*}$ & $\underset{\substack{\hat{S} \\
\delta}}{ }$ & $\begin{array}{l}\stackrel{\infty}{0} \\
\stackrel{0}{0}\end{array}$ & & प艹 & $\underset{\stackrel{\sim}{N}}{\stackrel{\sim}{\delta}}$ & $\underset{\widetilde{N}}{\stackrel{0}{-}}$ & 目 & 舍 &  & $\underset{m}{\stackrel{m}{m}}$ & $\stackrel{\circ}{\circ}$ \\
\hline 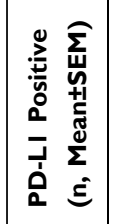 & $\underline{m} \simeq$ & $\bar{N} \sigma$ & $\underline{\underline{n}}$ & 으 &  & $\sigma \stackrel{0}{0}$ & $\underline{m} \simeq$ & $\underline{\circ}$ & 으느 & $0=$ & $\circ \underline{ }$ \\
\hline 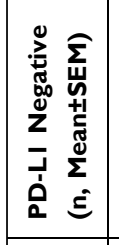 & $n=$ & \pm & $\simeq$ & $\circ$ & 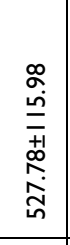 & $\underline{\circ} \infty$ & $\stackrel{0}{\infty}$ & $=n$ & 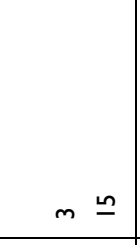 & $\sim \subseteq$ & $\infty \infty N$ \\
\hline & 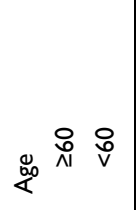 &  & 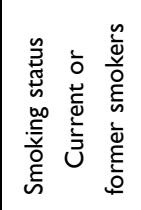 & 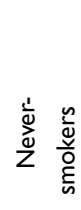 & 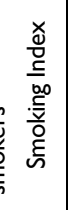 & 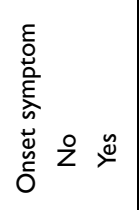 &  & 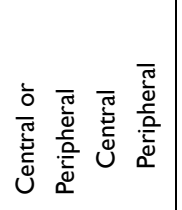 & 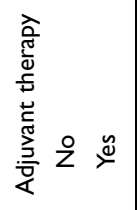 & 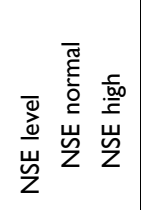 & 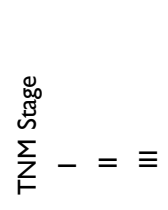 \\
\hline
\end{tabular}



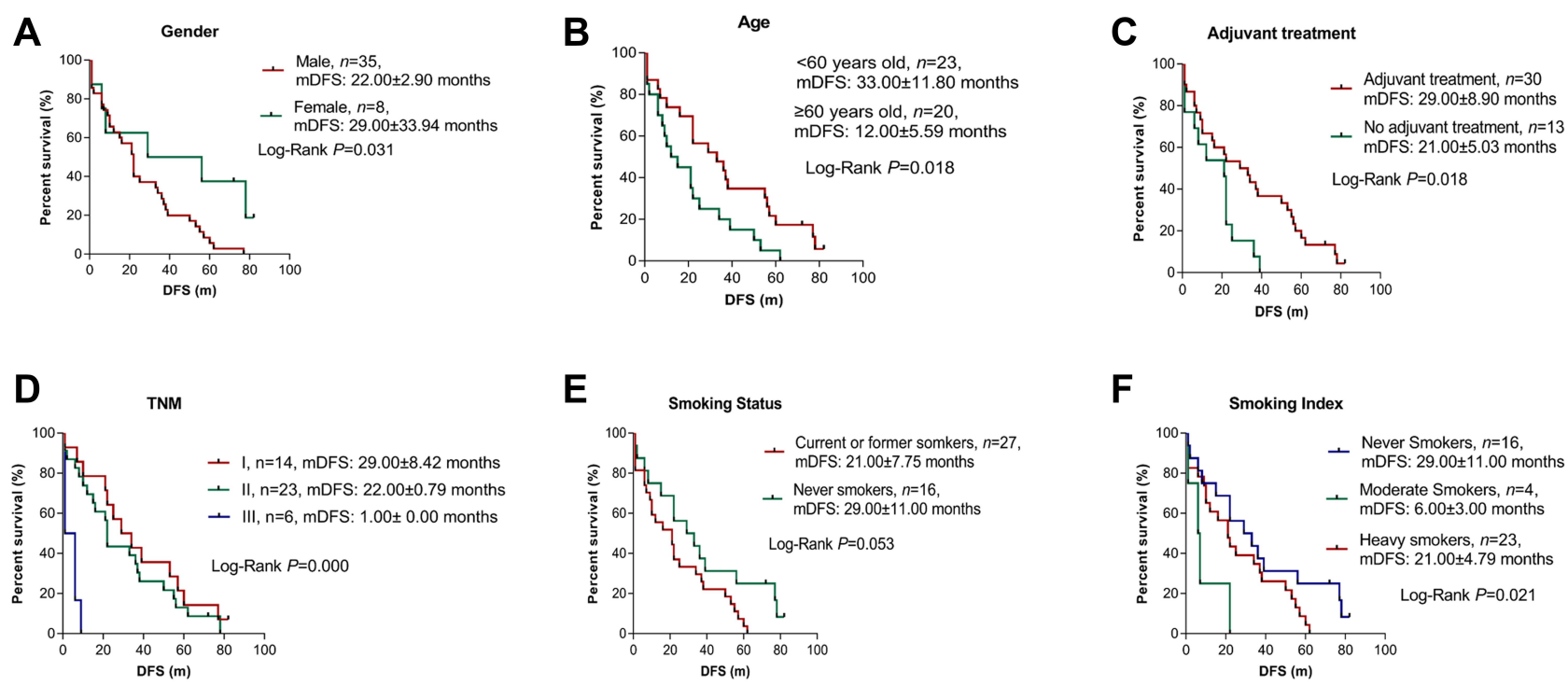

Figure 2 Clinicalpathological factors including gender (A), age (B), adjuvant treatment (C), TNM stage (D), smoking status (E), smoking index (F) and DFS.


Figure 3 The correlation between PD-LI (A), PARPI (B), DDL-3 (C) and DFS.

\section{Adjuvant Treatment, TNM Stage, and PD-LI Expression Were Associated with OS}

Patients who did not receive adjuvant therapy (either chemotherapy or radiotherapy) showed worse OS than those who did (mOS: $37.00 \pm 9.14$ vs $22.00 \pm 11.27$ months in patients with or without adjuvant therapy, respectively, $P=0.002$, Figure 4A). Advanced TNM stage also correlated with poor prognosis (mOS: $39.00 \pm 0.00,32.00 \pm 6.39$, and $6.00 \pm 2.89$ months in TNM stage I-III, respectively, $P=0.003$, Figure 4B). Other clinicopathological factors, such as age, gender, smoking status, central or peripheral tumor, comorbidities, onset symptoms, and NSE level did show a significant difference in OS (Supplementary Figure 4A-G).

Patients with positive PD-L1 expression had significantly worse OS than those with negative PD-L1 (mOS: $22.00 \pm 7.49$ vs NR, $P=0.000$; Figure $4 \mathrm{C}$ ). However, neither DLL-3, PAPR1, ASCL-1, or SLFN11 had a significant effect on OS (Figure 4D and E, Supplementary Figure $\underline{3 \mathrm{~B}}$ and $\underline{\mathrm{D}}$.

Univariate analysis revealed that PD-L1 and TNM stage were significantly associated with poor $\mathrm{OS}(\mathrm{HR}=4.40,95 \%$ $\mathrm{CI}=1.87-10.37, P=0.001 ; \mathrm{HR}=2.38,95 \% \mathrm{CI}=1.30-4.33$, $P=0.005$ for PD-L1 and TNM stage, respectively), whereas adjuvant treatment was significantly correlated with favorable OS $(\mathrm{HR}=0.33,95 \% \mathrm{CI}=0.16-0.70, P=0.004)$. Further multivariate analysis indicated that PD-L1 and TNM stage were independent and significant negative predictive factors for $\mathrm{OS}(\mathrm{HR}=2.89,95 \% \mathrm{CI}=1.21-6.93, P=0.017 ; \mathrm{HR}=2.49$, 95\% $\mathrm{CI}=1.25-4.94, P=0.009$ for PD-L1 and TNM stage, respectively), and adjuvant treatment was an independent positive prognostic factor for $\mathrm{OS} \quad(\mathrm{HR}=0.37,95 \%$ $\mathrm{CI}=0.17-0.81, P=0.012$, Table 4).

\section{Discussion}

Recently, novel targets, such as PD-1/PD-L1 antibodies, ${ }^{14}$ PARP, and DLL-3 inhibitors have revolutionized the 
Table 3 Univariate and Multivariate Analyses of Relationship Between DFS and PD-LI and Clinicopathological Factors

\begin{tabular}{|c|c|c|c|c|}
\hline \multirow[t]{2}{*}{ Variable } & \multicolumn{2}{|c|}{ Univariate Analysis } & \multicolumn{2}{|c|}{ Multivariate Analysis } \\
\hline & HR $(95.0 \% \mathrm{Cl})$ & $\mathbf{P}$ & HR $(95.0 \% \mathrm{Cl})$ & $\mathbf{P}$ \\
\hline $\begin{array}{l}\text { Gender } \\
\text { (Female vs Male) }\end{array}$ & $0.36(0.14-0.96)$ & 0.040 & $0.45(0.13-1.53)$ & 0.203 \\
\hline $\begin{array}{l}\text { Age } \\
\text { ( } \geq 60 \text { years old } v s<60 \text { years old })\end{array}$ & $2.11(1.11-4.03)$ & 0.024 & $1.43(0.69-3.00)$ & 0.339 \\
\hline $\begin{array}{l}\text { Smoking } \\
\text { (Current or former smokers vs Never smokers) }\end{array}$ & $1.93(0.97-3.85)$ & 0.063 & $1.26(0.52-3.09)$ & 0.610 \\
\hline Adjuvant therapy (Adjuvant therapy vs No adjuvant therapy) & $0.44(0.21-0.90)$ & 0.025 & $0.48(0.22-1.06)$ & 0.069 \\
\hline $\begin{array}{l}\text { TNM stage } \\
\text { (I vs II vs III) }\end{array}$ & $2.22(1.21-4.07)$ & 0.010 & $2.51(1.31-4.78)$ & 0.005 \\
\hline $\begin{array}{l}\text { PD-LI expression } \\
\text { (Positive vs Negative) }\end{array}$ & $1.88(0.98-3.60)$ & 0.057 & $1.09(0.54-2.19)$ & 0.810 \\
\hline
\end{tabular}

treatment of ES-SCLC, and the expression of PD-L1, DLL-3/ASCL-1, and PARP1/SLFN11 have been recognized as predictive markers for clinical response. However, the studies of immunotherapy or targetimmunotherapy in LS-SCLC are still on-going and the results are immature. The expression and prognostic values of PD-L1, PARP1, and DLL-3 are elusive in surgically resected LS-SCLC.

In this study, we enrolled 43 surgically resected LSSCLC patients and defined PD-L1 $>5 \%$ on both tumor cells and stromal cells as positive. We firstly examined the expression pattern of PD-L1, PARP1, and DLL-3 in specimen from surgically resected LS-SCLC. PD-L1 expression in SCLC varied from one study to another. ${ }^{10,15,16}$ After scrutinizing the data, we suppose reasons that led to different prevalence of PD-L1 are as follows: firstly, there is no standard for positivity of PD-L1 expression, and a cutoff of at least $1 \%$ or $5 \%$ cells staining were both defined as positive. Early in 2016, Takada et al ${ }^{17}$ concluded that $5 \%$ cut-off showed similar rates of expression among antibodies clones E1L3N, 28-8, and SP142. Herein, we decided to use 5\% cut-off in this study. Secondly, PD-L1 is low-expressed in tumor cells but highly expressed in tumor infiltrating immune cells. When taking the expression of PD-L1 in tumor infiltrating immune cells
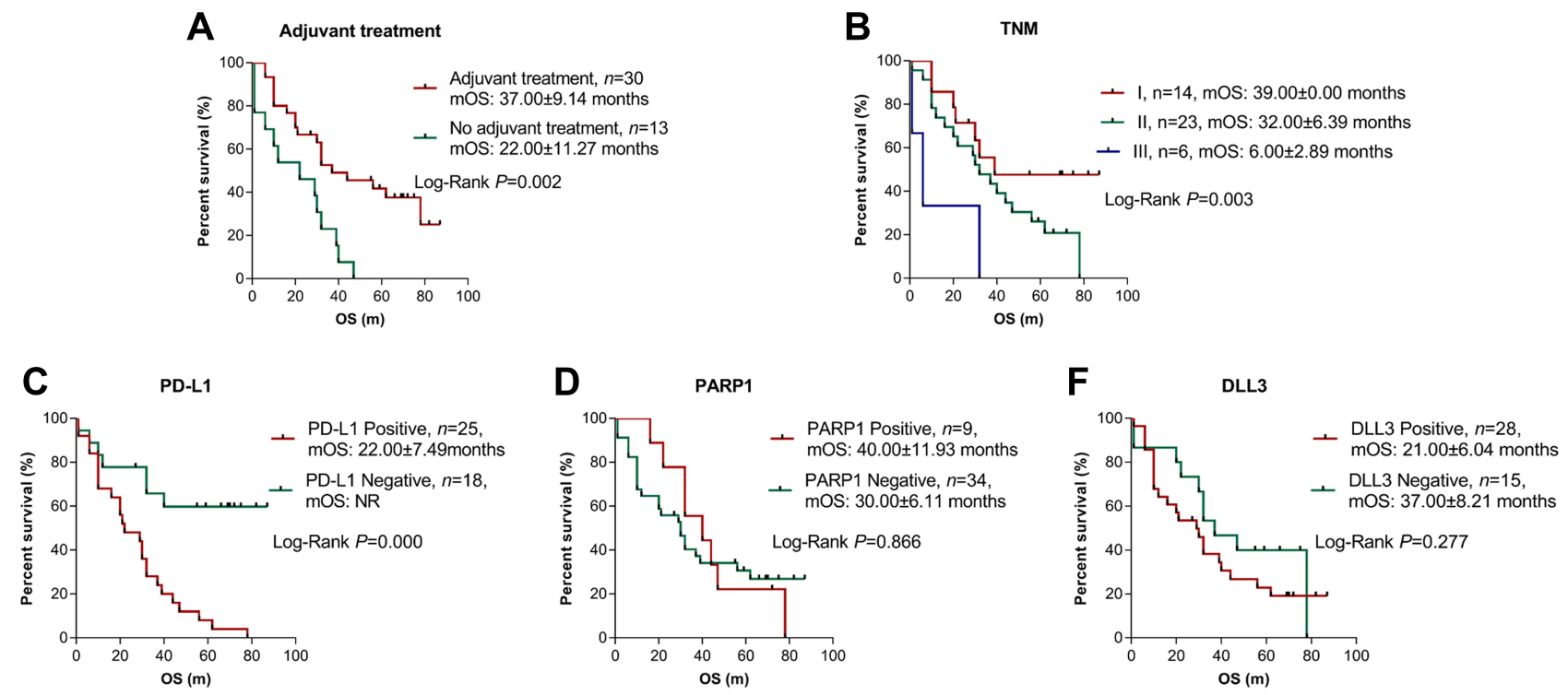

Figure 4 The correlation between Adjuvant treatment (A), TNM stage (B), PD-LI (C), PARPI (D), DDL-3 (E) and OS. 
Table 4 Univariate and Multivariate Analyses of Relationship Between OS and PD-Lland Clinicopathological Factors

\begin{tabular}{|l|l|l|l|l|}
\hline \multirow{2}{*}{ Variables } & \multicolumn{2}{l|}{ Univariate Analysis } & \multicolumn{2}{l|}{ Multivariate Analysis } \\
\cline { 2 - 5 } & HR (95.0\% CI) & $\mathbf{P}$ & HR (95.0\% CI) \\
\hline $\begin{array}{l}\text { Adjuvant therapy } \\
\text { (Adjuvant therapy vs No adjuvant therapy) }\end{array}$ & $4.40(1.87-10.37)$ & 0.004 & $0.37(0.17-0.81)$ & $\mathbf{P}$ \\
\hline $\begin{array}{l}\text { TNM stage } \\
\text { (I vs II vs III) }\end{array}$ & $0.33(0.16-0.70)$ & 0.005 & $2.49(1.25-4.94)$ \\
\hline $\begin{array}{l}\text { PD-LI expression } \\
\text { (Positive vs Negative) }\end{array}$ & $2.38(1.30-4.33)$ & 0.001 & $2.89(1.21-6.93)$ & 0.009 \\
\hline
\end{tabular}

into account, the prevalence of PD-L1 increased from 14.7\% to $57.1 \%$. Our results were similar to Ishii et al's, ${ }^{10}$ in which PD-L1 expression on both tumor cells and tumor infiltrating immune cells were measured. Thirdly, different antibodies used in these studies also led to varied prevalence of PD-L1. Antibodies from Abcam achieved the highest positive rate, whereas none of the tumor cells were positive for PD-L1 staining by antibody 5H1/Chen's lab. ${ }^{18}$ The positivity of other antibodies such as 28-8, ${ }^{19} 22 \mathrm{C} 3 / \mathrm{Dako},{ }^{20,21} \mathrm{E} 1 \mathrm{~L} 3 \mathrm{~N} /$ $\mathrm{CST}^{18}{ }^{18}$ and MAB1561/R\&D system ${ }^{22}$ varied from $10-34.4 \%$ in tumor cells, and from $2-87.1 \%$ in both tumor and stromal cells. Most recently, Takayama et $\mathrm{al}^{23}$ compared different PD-L1 antibodies again and came to the conclusion that antiPD-L1 antibody clones (28-8, 22C3, and SP263) exhibited similar positive rate and with similar clinical correlates. Last, but not least, LS-SCLC and ES-SCLC exhibited different PD-L1 prevalence, and LS-SCLC seemed to have higher PDL1 expression, ${ }^{10,24}$ which explains the higher PD-L1 proportion in our cohort.

Besides PD-1 and PD-L1, DLL-3 and PARP1 inhibitors also gained promising data in Phase I and II clinical trials. We also observed high prevalence of DLL-3 and ASCL-1 in surgically resected LS-SCLC, as has been demonstrated in a Japanese cohort and a Chinese study. ${ }^{25}$ Contrary to previous studies, the positive expression rate was relatively low in our cohort. We suppose it is because we only included surgically resected LS-SCLC in our study.

We next identify the correlation between clincal pathlogical factors and PD-L1, PARP1, DLL-3, as well as the prognostic effects of clincal pathlogical factors. As cigarette smoking has been proved to lead to tumor spread, ${ }^{26,27}$ we noticed that current or former smokers did perform shorter DFS than never smokers, though it was not significantly different. Additionally, moderate smokers were proved to have the worst survival, which may be due to a limited number of moderate smokers in our cohort.

Although systemic chemotherapy using cisplatin/etoposide with concurrent thoracic radiation is a standard treatment for $\mathrm{LS}-S C L C^{28}$ locoregional relapse is still frequent $(26-63 \%) .{ }^{29,30}$ Masayoshi Inoue et $\mathrm{al}^{31}$ reviewed several clinical trials and concluded that surgery reduced the locoregional relapse rate in LS-SCLC. Moreover, perioperative chemotherapy has also been proved to prolong survival. ${ }^{32}$ In accordance with these studies, we also demonstrated that adjuvant treatment prolonged LS-SCLC patients' DFS and OS.

Interestingly, we identified high PD-L1 correlated with shorter DFS and OS and was an individual negative prognostic factor in surgically resected LS-SCLC for OS, in accordance with two studies from China. ${ }^{33,34}$ However, the prognostic value of PD-L1 in SCLC is controversial: in contrast to our findings, two Japanese cohorts ${ }^{10,35}$ demonstrated that PD-L1 correlated with better prognosis. In resectable SCLC, positive PD-L1 expression predicted improved DFS and a lower risk of brain metastasis. ${ }^{36}$ Moreover, another study conducted among 66 stage I-III SCLC patients and 38 metastatic cases showed no effect of PD-L1 expression on OS. ${ }^{24}$ The prognostic role of PD-L1 still needs to be explored, especially in LS-SCLC.

Cell intrinsic PD1/PD-L1 signaling has been demonstrated as a predictor for poor efficacy of cisplatin treatment, ${ }^{37}$ and it explains that PD-L1 predicted poor prognosis in our study, in which we enrolled 30 patients receiving adjuvant treatment. Thus, targeting the cellular PD1/PD-L1 axis may improve chemosensitization of aggressive SCLC.

In conclusion, we found that PD-L1 expression, TNM stage, and adjuvant treatment were independent prognostic factors for OS in surgically resected LS-SCLC. Our findings provide evidence for adjuvant treatment, whether chemotherapy or radiotherapy, in surgically resected LS-SCLC. 
Additionally, PD-1/PD-L1 antibodies may improve survival for surgically resected LS-SCLC patients, and further perspective studies are needed.

Here are several limitations of our study: firstly, the number of cases is limited. Since SCLC is a highly aggressive neuroendocrine tumor, characterized by rapid growth and early tendency to widespread metastasis, over $70 \%$ of new diagnosed cases were already metastatic. In our study, the percentage of LS-SCLC patients is relatively low $(13.86 \%$; 56/404). Secondly, none of the patients received immunotherapy or DLL-3/PAPR1 inhibitors in our cohort, so we could not analyze the correlation between PD-L1, DLL-3, PARP1, and immunotherapy or targeted therapy efficacy. Thirdly, since we did not obtain adequate tumor tissues from ES-SCLC biopsy samples, we could not compare the expression of PD-L1, PARP1, and DLL-3 between LS-SCLC and ES-SCLC, and we would love to conduct a perspective study in the near future.

\section{Conclusion}

PD-L1 and DLL-3 were highly-expressed in SCLC. PD$\mathrm{L} 1$, adjuvant treatment, and TNM stage were individual prognostic factors for OS in surgically resected LS-SCLC.

\section{Grant Support}

International cooperation project in science and technology of Shaanxi province (No. 2019KW-074).

\section{Acknowledgments}

We acknowledged all the patients and their families who participated in our research. We also acknowledged International cooperation project in science and technology of Shaanxi province (No. 2019KW-074) for supporting this research.

\section{Disclosure}

The authors disclose no potential conflicts of interest.

These authors share the senior authorship: Tao Tian and Yu Yao.

\section{References}

1. Reck M, Liu SV, Mansfield AS, et al. 1736OIMpower133: updated overall survival (OS) analysis of first-line (1L) atezolizumab (atezo) + carboplatin + etoposide in extensive-stage SCLC (ES-SCLC). Annals Oncol. 2019;30 (Supplement 5):v710v711. doi:10.1093/annonc/mdz264

2. Morimoto M, Nishinakamura R, Saga Y, Kopan R. Different assemblies of Notch receptors coordinate the distribution of the major bronchial Clara, ciliated and neuroendocrine cells. Development. 2012;139(23):4365-4373.
3. Sabari JK, Lok BH, Laird JH, Poirier JT, Rudin CM. Unravelling the biology of SCLC: implications for therapy. Nature Rev Clin Oncol. 2017;14(9):549-561. doi:10.1038/nrclinonc.2017.71

4. Saunders LR, Bankovich AJ, Anderson WC, et al. A DLL3-targeted antibody-drug conjugate eradicates high-grade pulmonary neuroendocrine tumor-initiating cells in vivo. Science Translational Medicine. 2015;7(302):302ra136. doi:10.1126/scitranslmed.aac9459

5. Rudin CM, Pietanza MC, Bauer TM, et al. Rovalpituzumab tesirine, a DLL3-targeted antibody-drug conjugate, in recurrent small-cell lung cancer: a first-in-human, first-in-class, open-label, Phase 1 study. Lancet Oncol. 2017;18(1):42-51. doi:10.1016/S14702045(16)30565-4

6. Atrafi F, Groen HJM, Byers LA, et al. A phase i dose-escalation study of veliparib combined with carboplatin and etoposide in patients with extensive-stage small cell lung cancer and other solid tumors. Clin Cancer Res. 2019;25(2):496-505. doi:10.1158/10780432.CCR-18-2014

7. Owonikoko TK, Dahlberg SE, Khan SA, et al. A phase 1 safety study of veliparib combined with cisplatin and etoposide in extensive stage small cell lung cancer: A trial of the ECOG-ACRIN cancer research group (E2511). Lung Cancer. 2015;89(1):66-70.

8. Pietanza MC, Waqar SN, Krug LM, et al. Randomized, double-blind, phase ii study of temozolomide in combination with either veliparib or placebo in patients with relapsed-sensitive or refractory small-cell lung cancer. J Clin Oncol. 2018;36(23):2386-2394. doi:10.1200/ JCO.2018.77.7672

9. Tian T, Nan K-J, Wang S-H, et al. PTEN regulates angiogenesis and VEGF expression through phosphatase-dependent and -independent mechanisms in HepG2 cells. Carcinogenesis. 2010;31(7):1211-1219. doi:10.1093/carcin/bgq085

10. Ishii H, Azuma K, Kawahara A, et al. Significance of programmed cell death-ligand 1 expression and its association with survival in patients with small cell lung cancer. $J$ Thoracic Oncol. 2015;10 (3):426-430. doi:10.1097/JTO.0000000000000414

11. Mu Y, Lou J, Srivastava M, et al. SLFN11 inhibits checkpoint maintenance and homologous recombination repair. EMBO Rep. 2016;17(1):94-109. doi:10.15252/embr.201540964

12. Murai J, Feng Y, Yu GK, et al. Resistance to PARP inhibitors by SLFN11 inactivation can be overcome by ATR inhibition. Oncotarget. 2016;7(47):76534-76550.

13. Furuta M, Sakakibara-Konishi J, Kikuchi H, et al. Analysis of DLL3 and ASCL1 in surgically resected small cell lung cancer (HOT1702). oncologist. 2019;24(11):e1172-e1179. doi:10.1634/theoncologist.2018-0676

14. Pakkala S, Owonikoko TK. Immune checkpoint inhibitors in small cell lung cancer. $J$ Thorac Dis. 2018;10(Suppl 3):S460-s467. doi:10.21037/jtd.2017.12.51

15. Saito M, Saito K, Shiraishi K, et al. Identification of candidate responders for anti-PD-L1/PD-1 immunotherapy, Rova-T therapy, or EZH2 inhibitory therapy in small-cell lung cancer. Molecular Clin Oncol. 2018;8(2):310-314.

16. $\mathrm{Yu} \mathrm{H}$, Batenchuk $\mathrm{C}$, Badzio A, et al. PD-L1 expression by two complementary diagnostic assays and mRNA in situ hybridization in small cell lung cancer. J Thoracic Oncol. 2017;12(1):110-120. doi:10.1016/j.jtho.2016.09.002

17. Takada K, Toyokawa G, Okamoto T, et al. An Immunohistochemical analysis of PD-L1 protein expression in surgically resected small cell lung cancer using different antibodies and criteria. Anticancer Res. 2016;36(7):3409-3412.

18. Schultheis AM, Scheel AH, Ozretic L, et al. PD-L1 expression in small cell neuroendocrine carcinomas. European J Cancer. 2015;51 (3):421-426. doi:10.1016/j.ejca.2014.12.006

19. Antonia SJ, Lopez-Martin JA, Bendell J, et al. Nivolumab alone and nivolumab plus ipilimumab in recurrent small-cell lung cancer (CheckMate 032): a multicentre, open-label, phase 1/2 trial. Lancet Oncol. 2016;17(7):883-895. doi:10.1016/S1470-2045(16)30098-5 
20. Weiss GJ, Waypa J, Blaydorn L, et al. A phase Ib study of pembrolizumab plus chemotherapy in patients with advanced cancer (PembroPlus). Br J Cancer. 2017;117(1):33-40. doi:10.1038/ bjc. 2017.145

21. Ott PA, Elez E, Hiret S, et al. Pembrolizumab in patients with extensive-stage small-cell lung cancer: results from the phase Ib KEYNOTE-028 study. J Clin Oncol. 2017;35(34):3823-3829. doi:10.1200/JCO.2017.72.5069

22. Kim HS, Lee JH, Nam SJ, et al. Association of PD-L1 expression with tumor-infiltrating immune cells and mutation burden in high-grade neuroendocrine carcinoma of the lung. $J$ Thoracic Oncol. 2018;13(5):636-648.

23. Yoshimura A, Yamada T, Miyagawa-Hayashino A, et al. Comparing three different anti-PD-L1 antibodies for immunohistochemical evaluation of small cell lung cancer. Lung Cancer. 2019;137:108-112. doi:10.1016/j.lungcan.2019.09.012

24. Bonanno L, Pavan A, Dieci MV, et al. The role of immune microenvironment in small-cell lung cancer: distribution of PD-L1 expression and prognostic role of FOXP3-positive tumour infiltrating lymphocytes. European J Cancer. 2018;101::191-200. doi:10.1016/ j.ejca.2018.06.023

25. Yan LX, Liu YH, Li Z, et al. Prognostic value of delta-like protein 3 combined with thyroid transcription factor-1 in small-cell lung cancer. Oncol Lett. 2019;18(3):2254-2261.

26. Pesch B, Kendzia B, Gustavsson P, et al. Cigarette smoking and lung cancer-relative risk estimates for the major histological types from a pooled analysis of case-control studies. Int $j$ Cancer. 2012;131 (5):1210-1219. doi:10.1002/ijc.27339

27. Pezzuto A, Citarella F, Croghan I, Tonini G. The effects of cigarette smoking extracts on cell cycle and tumor spread: novel evidence. Future Science OA. 2019;5(5):Fso394. doi:10.2144/ fsoa-2019-0017

28. Zimmermann FB, Bamberg M, Molls M, Jeremic B. Limited-disease small-cell lung cancer. Semin Surg Oncol. 2003;21(3):156-163. doi:10.1002/ssu. 10033
29. Murray N, Coy P, Pater JL, et al. Importance of timing for thoracic irradiation in the combined modality treatment of limited-stage small-cell lung cancer. The national cancer institute of canada clinical trials group. J clin oncol. 1993;11(2):336-344.

30. Jeremic B, Shibamoto Y, Acimovic L, Milisavljevic S. Initial versus delayed accelerated hyperfractionated radiation therapy and concurrent chemotherapy in limited small-cell lung cancer: a randomized study. J clin oncol. 1997;15(3):893-900. doi:10.1200/JCO.1997.15.3.893

31. Inoue M, Sawabata N, Okumura M. Surgical intervention for small-cell lung cancer: what is the surgical role? Gen Thorac Cardiovasc Surg. 2012;60(7):401-405. doi:10.1007/s11748-012-0072-9

32. Yang CF, Chan DY, Speicher PJ, et al. Role of adjuvant therapy in a population-based cohort of patients with early-stage small-cell lung cancer. J Clin Oncol. 2016;34(10):1057-1064. doi:10.1200/ JCO.2015.63.8171

33. Chang YL, Yang CY, Huang YL, Wu CT, Yang PC, High PD. L1 expression is associated with stage IV disease and poorer overall survival in 186 cases of small cell lung cancers. Oncotarget. 2017;8 (11):18021-18030. doi:10.18632/oncotarget.14935

34. Qiu YJ, Zhang MZ. [PD-L1 expression is related with prognosis of small cell lung cancer]. Zhonghua Zhong liu Za Zhi. 2019;41 (3):214-217. Chinese.

35. Toyokawa G, Takada K, Haratake N, et al. Favorable disease-free survival associated with programmed death ligand 1 expression in patients with surgically resected small-cell lung cancer. Anticancer Res. 2016;36(8):4329-4336.

36. Liu J, Lu Z, Wang W, Sun X. Programmed death-ligand 1 positivity can predict improved survival and a lower risk of brain metastasis in patients with resectable small cell lung cancer. Oncol Lett. 2018;16 (2):2373-2381.

37. Yan F, Pang J, Peng Y, Molina JR, Yang P, Liu S. Elevated cellular PD1/PD-L1 expression confers acquired resistance to cisplatin in small cell lung cancer cells. PLoS One. 2016;11(9):e0162925. doi:10.1371/journal.pone.0162925

\section{Publish your work in this journal}

Cancer Management and Research is an international, peer-reviewed open access journal focusing on cancer research and the optimal use of preventative and integrated treatment interventions to achieve improved outcomes, enhanced survival and quality of life for the cancer patient.
The manuscript management system is completely online and includes a very quick and fair peer-review system, which is all easy to use. Visit http://www.dovepress.com/testimonials.php to read real quotes from published authors. 